\title{
On three Parameter Weighted Quasi Lindley Distribution: Properties and Applications
}

\author{
${ }^{1 *}$ Anwar Hassan, ${ }^{2}$ Sameer Ahmad Wani and ${ }^{3}$ Bilal Ahmad Para \\ ${ }^{1,2}$ Department of Statistics, University of Kashmir, Srinagar, India \\ ${ }^{3}$ Department of Statistics, Government Degree College, Anantnag, India \\ *Corresponding Author: anwar.hassan5@gmail.com
}

Available online at: www.isroset.org

Received: 09/Sept./2018, Accepted 13/Oct/2018, Online 31/Oct/2018

Abstract- In this paper, we have introduced a weighted model of the Quasi Lindley Distribution (QLD) as a new generalization of QLD. Statistical properties of this new distribution are derived and the model parameters are estimated by Maximum Likelihood (ML) estimation technique. Finally, the model is examined with an application to real life data.

Keywords: Quasi Lindley Distribution, Weighted Probability Models, Reliability and Order Statistics, Strength Data.

\section{INTRODUCTION}

Shanker and Mishra (2013) introduced a two-parameter Quasi Lindley distribution (QLD), of which the Lindley distribution (LD) is a particular case. Its moments, failure rate function, mean residual life function and stochastic orderings have been discussed.

Probability density function (pdf) of Quasi Lindley Distribution (QLD) is given by

$f(x ; \alpha, \theta)=\frac{\theta(\alpha+\theta x)}{\alpha+1} e^{-\theta x} \quad x>0, \alpha>0, \theta>0$

The corresponding cdf of (1.1) is given by

$$
F(x ; \alpha, \theta)=1-\left[\frac{1+\alpha+\theta x}{\alpha+1}\right] e^{-\theta x}, x>0, \theta>0
$$

The rth moment of (1.1) is given as

$\mu_{r}^{\prime}=\frac{(\alpha+r+1) \Gamma(r+1)}{\theta^{r}(\alpha+1)}, r=1,2,3, \ldots$

\section{WEIGHTED QUASI LINDLEY DISTRIBUTION (WQLD)}

Weighted technique is one of the prominent techniques for generalizing the probability models using the weight functions. The concept of weighted distributions can be traced to the work of Fisher (1934), in connection with his studies, on how methods of ascertainment can influence the form of distribution of recorded observations. Later it was introduced and formulated in general terms by Rao (1965). Many researchers developed some important weighted probability models with their significant role in handling data sets from various practical fields. Gove (2003) studied some of the more recent results on weighted distributions pertaining to parameter estimation in forestry. Gupta and Tripathi (1996) studied the weighted version of the bivariate three parameter logarithmic series distribution which has applications in many fields such as ecology, social and behavioral sciences and species abundance studies. Warren (1975) was the first to apply the weighted distributions in 
connection with sampling wood cells. Recently Para and jan (2018) introduced weighted Pareto type II distribution with applications in medical sciences.

If $\mathrm{X}$ is a non negative random variable with probability density function (pdf) $f(x)$. Let $w(x)$ be the weight function which is a non negative function, then the probability density function of the weighted random variable $X_{w}$ is given by:

$$
f_{w}(x)=\frac{w(x) f(x)}{E(w(x))}, \quad x>0,
$$

where $w(x)$ be a non-negative weight function and $E(w(x))=\int w(x) f(x) d x<\infty$.

In this paper, we have considered the weight function as $w(x)=x^{c}$ to obtain the weighted Quasi Lindley distribution.

The probability density function (pdf) of weighted Quasi Lindley distribution is given as:

$f_{w}(x ; c, \alpha, \theta)=\frac{x^{c} f(x, \theta)}{E\left[x^{c}\right]}$,

$f_{w}(x ; c, \alpha, \theta)=\frac{x^{c} \theta^{(c+1)}(\alpha+\theta x) e^{-\theta x}}{(\alpha+c+1) \Gamma(c+1)}, \quad x>0, c>0, \alpha>0, \theta>0$,

where $E\left(x^{c}\right)=\frac{(\alpha+c+1) \Gamma(r+1)}{\theta^{c}(\alpha+1)}$.

The corresponding cumulative distribution function (cdf) of weighted Quasi Lindley Distribution (WQLD) is obtained as

$$
\begin{aligned}
F_{w}(x ; c, \alpha, \theta) & =\int_{0}^{x} f_{w}(x ; c, \alpha, \theta) d x \\
& =\int_{0}^{x} \frac{x^{c} \theta^{(c+1)}(\alpha+\theta x) e^{-\theta x}}{(\alpha+c+1) \Gamma(c+1)} d x \quad \text {, put } \theta x=t, \quad \theta d x=d t,
\end{aligned}
$$

as $x \rightarrow 0, t \rightarrow 0$ and $x \rightarrow x, t \rightarrow \theta x \quad$, after simplification

$F_{w}(x ; c, \alpha, \theta)=\frac{1}{(\alpha+c+1) \Gamma(c+1)}(\alpha \gamma(c+1, \theta x)+\gamma(c+2, \theta x)), \quad x>0, c>0, \alpha>0, \theta>0$,

where $\theta$ and $c$ are positive parameters and $\gamma(s, x)=\int_{0}^{x} t^{s-1} e^{-t} d t$ is a lower incomplete gamma function.

The graphs of probability density function and cumulative distribution function are plotted for different values of parameters $\theta$ and $c$ given in Fig.1 and Fig. 2 respectively. Fig. 1 gives the description of some of the possible shapes of weighed Quasi Lindley distribution for different values of the parameters $\theta$ and $c$. It illustrates that the density function of weighted Quasi Lindley distribution is positively skewed, for fixed $\theta$ it becomes more and more flatter as the value of $c$ is increased. Fig. 2 shows the graph of distribution function which is an increasing function.

\section{SPECIAL CASES}

Case I: If we put $c=0$, then weighted Quasi Lindley distribution (2.1) reduces to Quasi Lindley distribution with probability density function as:

$f(x ; \alpha, \theta)=\frac{\theta(\alpha+\theta x)}{\alpha+1} e^{-\theta x} \quad x>0, \alpha>0, \theta>0$, 
Case II: For $\alpha=0$, then weighted Quasi Lindley distribution (2.1) reduces to two parameter gamma distribution with parameters $\theta$ and $c+2$.

Fig.1: pdf plot of Weighted Quasi Lindley Distribution

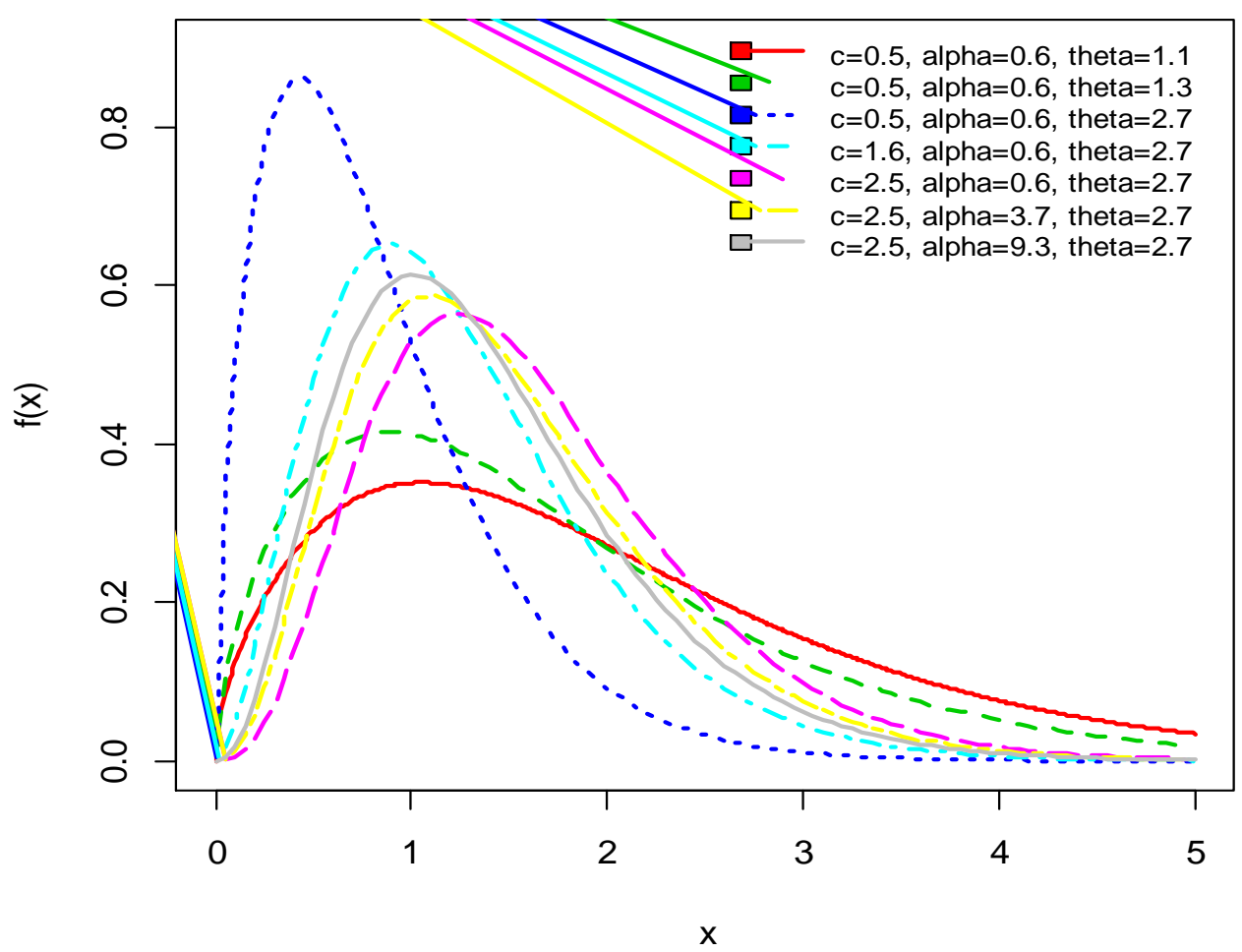

\section{RELIABILITY ANALYSIS}

In this section, we have obtained the reliability, hazard rate, reverse hazard rate of the proposed weighted Quasi Lindley Distribution.

\subsection{Reliability function $R(x)$}

The reliability function is defined as the probability that a system survives beyond a specified time. It is also referred to as survival or survivor function of the distribution. It can be computed as complement of the cumulative distribution function of the model. The reliability function or the survival function of weighted Quasi Lindley distribution is calculated as:

$R_{w}(x, c, \theta)=1-\frac{1}{(\alpha+c+1) \Gamma(c+1)}(\alpha \gamma(c+1, \theta x)+\gamma(c+2, \theta x)), \quad x>0, c>0, \alpha>0, \theta>0$,

The graphical representation of the reliability function for the weighted Quasi Lindley distribution is shown in fig. 3.

\subsection{Hazard Function:}

The hazard function is also known as hazard rate, instantaneous failure rate or force of mortality is given as:

$H . R=h(x ; c, \theta)=\frac{f_{w}(x, \theta)}{R_{w}(x, \theta)}=\frac{x^{c} \theta^{c+1}(\alpha+x \theta) e^{-\theta x}}{(\alpha+c+1) \Gamma(c+1)-\{\alpha \gamma(c+1, \theta x)+\gamma(c+2, \theta x)\}}, \quad x>0, c>0, \alpha>0, \theta>0$, 
Fig.2: CDF plot of Weighted Quasi Lindley Distribution

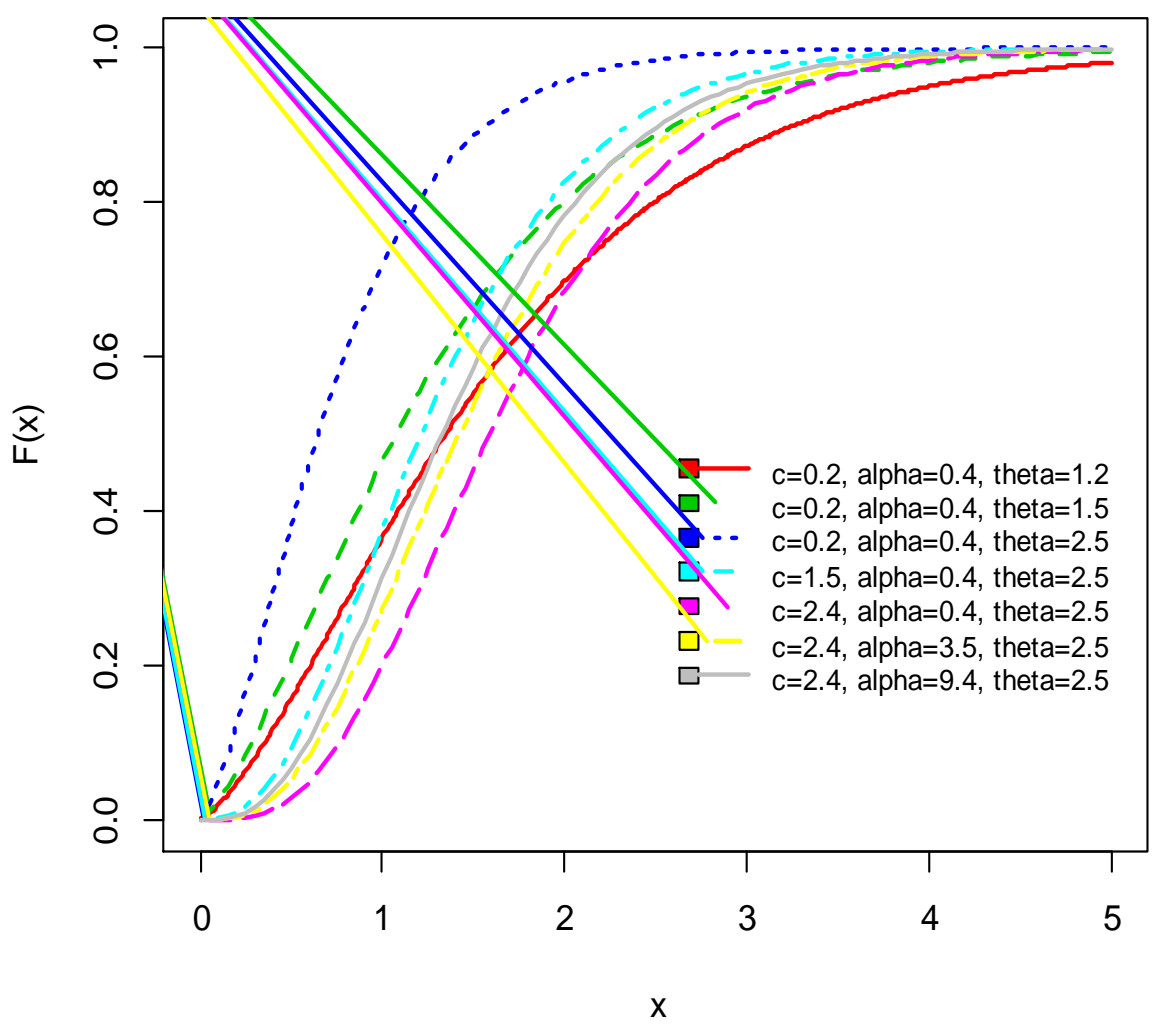

4.3 Reverse Hazard Rate:

The reverse hazard rate of the weighted quasi Lindley distribution are respectively given as:

R.H.R $=h_{r}(x, c, \theta)=\frac{f_{w}(x, \theta)}{F_{w}(x, \theta)}=\frac{x^{c} \theta^{c+1}(\alpha+\theta x) e^{-\theta x}}{(\alpha \gamma(c+1, \theta x)+\gamma(c+2, \theta x)} . \quad x>0, \theta>0, c>0$.

\section{STATISTICAL PROPERTIES}

In this section, the different structural properties of the proposed weighted quasi Lindley model have been evaluated. These include moments, mode, harmonic mean, moment generating function and characteristic function.

5.1 Moments: Suppose $\mathrm{X}$ is a random variable following weighted Quasi Lindley distribution with parameter $\theta$, and then the rth moment for a given probability distribution is given by

$$
\begin{aligned}
\mu_{r}^{\prime}=E\left(X_{w}{ }^{r}\right) & =\int_{0}^{\infty} x^{r} f_{w}(x, c, \theta) d x \\
& =\int_{0}^{\infty} x^{r} \frac{x^{c} \theta^{c+1}(\alpha+\theta x) e^{-\theta x}}{(\alpha+c+1) \Gamma(c+1)} d x
\end{aligned}
$$


$\mu_{r}^{\prime}=\frac{(c+r) !(\alpha+c+r+1)}{\theta^{r}(\alpha+c+1) \Gamma(c+1)}$

$\mu_{1}^{\prime}=\frac{(c+1)\left(\theta^{3}+(c+2)(c+3)\right)}{\left(\theta^{3}+(c+1)(c+2)\right.}$.

Which is mean of the weighted quasi Lindley distribution

Put $\mathrm{r}=2$ in equation (5.1) we get

$\mu_{2}^{\prime}=\frac{(c+1)(c+2)(\alpha+c+3)}{\theta^{2}(\alpha+c+1)}$

And variance of weighted quasi Lindley distribution is

$$
\begin{aligned}
V(X)= & \frac{(c+1)(c+2)(\alpha+c+3)}{\theta^{2}(\alpha+c+1)}-\frac{(c+1)^{2}(\alpha+c+2)^{2}}{\theta^{2}(\alpha+c+1)^{2}} \\
& =\frac{(c+1)}{\theta^{2}(\alpha+c+1)^{2}}\left\{(\alpha+c+1)(c+2)(\alpha+c+3)-(c+1)(\alpha+c+2)^{2}\right\}
\end{aligned}
$$

Fig.3: Survival function plot of Weighted Quasi Lindley Distribution

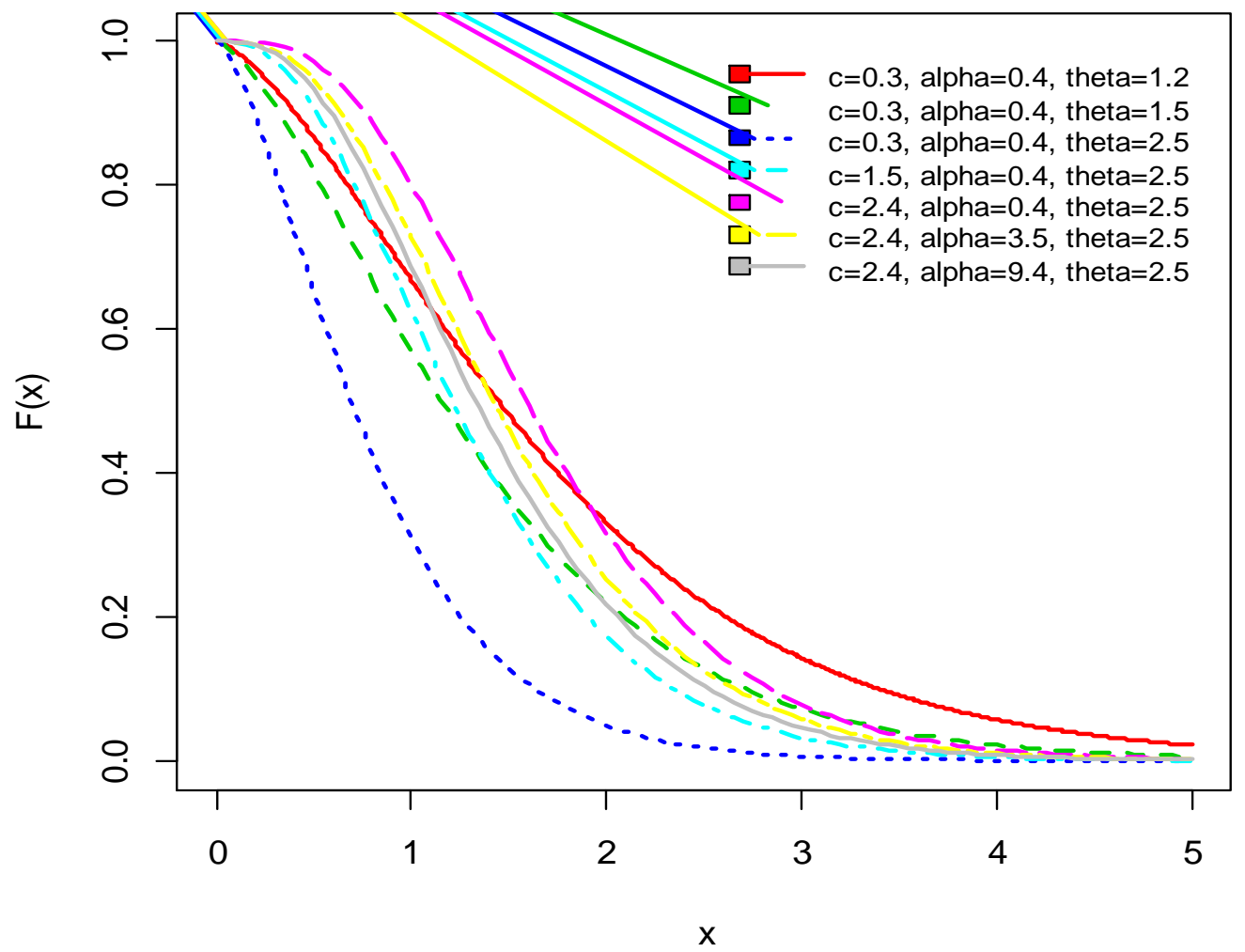




\subsection{Harmonic mean}

The harmonic mean for the proposed model is computed as:

$$
\begin{aligned}
H . M & =E\left[\frac{1}{X}\right]=\int_{0}^{\infty} \frac{1}{x} f_{w}(x ; c, \theta) d x \\
& =\int_{0}^{\infty} \frac{1}{x} \frac{x^{c} \theta^{c+1}(\alpha+x \theta) e^{-\theta x}}{(\alpha+c+1) \Gamma(c+1)} d x \\
& =\frac{c(\alpha+c+1)}{\theta(c+\alpha)}, \quad \theta>0, c>0,
\end{aligned}
$$

\subsection{Moment Generating Function and Characteristic Function of Weighted Quasi Lindley Distribution (WQLD)}

We will derive moment generating function and characteristic function of WQLD in this section.

Theorem 1.1: If $X$ has the $\operatorname{WQLD}(c, \theta, \alpha)$, then the moment generating function $M_{X}(t)$ and the characteristic function $\psi_{X}(t)$ are $\frac{\theta^{c+1}[\alpha(\theta-t)+\theta(c+1)]}{(\theta-t)^{c+2}(\alpha+c+1)}$ and $\frac{\theta^{c+1}[\alpha(\theta-i t)+\theta(c+1)]}{(\theta-i t)^{c+2}(\alpha+c+1)}$ respectively.

And hence show that two parameter gamma distribution is a particular case of weighted quasi Lindley distribution.

Proof: We begin with the well known definition of the moment generating function given by

$$
\begin{aligned}
M_{X}(t) & =E\left(e^{t x}\right)=\int_{0}^{\infty} e^{t x} f(x ; \alpha, \lambda) d x \\
& =\int_{0}^{\infty} \frac{e^{t x} x^{c} \theta^{c+1}(\alpha+\theta x) e^{-\theta x}}{(\alpha+c+1) \Gamma(c+1)} d x \\
& =\frac{\theta^{c+1}}{(\alpha+c+1) \Gamma(c+1)} \theta^{c+1} \int_{0}^{\infty} e^{-x(\theta-t)} x^{c+1-1}(\alpha+\theta x) d x \\
\Rightarrow M_{X}(t) & =\frac{\theta^{c+1}[\alpha(\theta-t)+\theta(c+1)]}{(\theta-t)^{c+2}(\alpha+c+1)}
\end{aligned}
$$

For $\alpha=0$ in equation (5.3.1) we get

$M_{X}(t)=\left[\frac{\theta}{(\theta-t)}\right]^{c+2}$

Which is mgf of two parameter gamma distribution with parameters $\theta$ and $\mathrm{c}+2$.

Also we know that $\psi_{X}(t)=M_{X}(i t)$

Therefore, $\psi_{X}(t)=\frac{\theta^{c+1}[\alpha(\theta-i t)+\theta(c+1)]}{(\theta-i t)^{c+2}(\alpha+c+1)}$ 


\subsection{Quantile and Random Number Generation from WQLD}

Inverse CDF Method is one of the methods used for the generation of random numbers from a particular distribution. In this method the random numbers from a particular distribution are generated by solving the equation obtained on equating the CDF of a distribution to a number $u$. The number $u$ is itself being generated from $U(0,1)$. Thus following the same procedure for the generation of random numbers from the WQLD we will proceed as

$F_{w}(x ; c, \alpha, \theta)=u$

$\frac{1}{(\alpha+c+1) \Gamma(c+1)} \alpha \gamma(c+1, \theta x)+\gamma(c+2, \theta x)=u$

On solving the equation (5.4.1) for $x$, we will obtain the required random number from the WQLD. Main problem, which is being faced while using this method of generating the random numbers is to solve the equations which are usually complex and complicated. In order to overcome such hindrance, we use softwares like MATLAB, Mathematica or R for solving such a complex equation.

\section{ORDER STATISTICS}

Let $X_{(1)}, X_{(2)}, X_{(3)} \ldots, X_{(n)}$ be the ordered statistics of the random sample $X_{1}, X_{2}, X_{3}, \ldots X_{n}$ drawn from the continuous distribution with cumulative distribution function $F_{X}(x)$ and probability density function $f_{X}(x)$, then the probability density function of rth order statistics $X_{(r)}$ is given by:

$f_{x(r)}(x, c, \theta)=\frac{n !}{(r-1) !(n-r) !} f(x)[F(x)]^{r-1}[1-F(x)]^{n-r} . \quad r=1,2,3, \ldots, n$

Using the equations (2.1) and (2.2), the probability density function of rth order statistics of weighted quasi Lindley distribution is given by:

$$
\begin{aligned}
& f_{w(r)}(x, c, \theta)=\frac{n !}{(r-1) !(n-r) !} \frac{x^{c} \theta^{c+3}\left(\theta+x^{2}\right) e^{-\theta x}}{c !\left(\theta^{3}+(c+1)(c+2)\right)}\left[\frac{1}{(\alpha+c+1) \Gamma(c+1)}(\alpha \gamma(c+1, \theta x)+\gamma(c+2, \theta x))\right]^{r-1} \\
& {\left[1-\frac{1}{(\alpha+c+1) \Gamma(c+1)}(\alpha \gamma(c+1, \theta x)+\gamma(c+2, \theta x))\right]^{n-r} . }
\end{aligned}
$$

Then, the pdf of first order $X_{(1)}$ weighted quasi Lindley distribution is given by:

$$
f_{w(1)}(x, c, \theta)=n \frac{x^{c} \theta^{c+1}(\alpha+\theta x) e^{-\theta x}}{(\alpha+c+1) \Gamma(c+1)}\left[1-\frac{1}{(\alpha+c+1) \Gamma(c+1)}(\alpha \gamma(c+1, \theta x)+\gamma(c+2, \theta x))\right]^{n-1} \text {. }
$$

and the pdf of nth order $X_{(n)}$ weighted quasi Lindley model is given as:

$$
f_{w(n)}(x, c, \theta)=n \frac{x^{c} \theta^{c+1}(\alpha+x \theta) e^{-\theta x}}{(\alpha+c+1) \Gamma(c+1)}\left[\frac{1}{(\alpha+c+1) \Gamma(c+1)}(\alpha \gamma(c+1, \theta x)+\gamma(c+2, \theta x))\right]^{n-1} \text {. }
$$




\section{MAXIMUM LIKELIHOOD ESTIMATION OF WEIGHTED QUASI LINDLEY DISTRIBUTION}

Let $x_{1}, x_{2}, \ldots, x_{n}$ be the random sample of size $\mathrm{n}$ drawn from weighted Quasi Lindley Distribution and let $f_{i}$ be the observed frequency in the sample corresponding to $X=x_{i}(i=1,2, \ldots, n)$, such that $\sum f_{i}=n$, where $k$ is the largest observed value having non-zero frequency then the likelihood function of Weighted Quasi Lindley Distribution is given as:

$$
\begin{aligned}
& L(x \mid c, \theta, \alpha)=\prod_{i=1}^{n} f(x ; c, \theta)=\prod_{i=1}^{n} \frac{x^{c} \theta^{c+1}(\alpha+x \theta) e^{-\theta x}}{(\alpha+c+1) \Gamma(c+1)} \\
& L(x \mid c, \theta, \alpha)=\prod\left[\left(x_{i}\right)^{c f_{i}}\right]^{n(c+1)} e^{-n \theta \bar{x}} \prod\left[\left(\alpha+\theta x_{i}\right)^{f_{i}}\right] \frac{1}{(\Gamma(c+1))^{n}(\alpha+c+1)^{n}}
\end{aligned}
$$

The log likelihood function becomes:

$$
\left.\log L(x \mid c, \theta, \alpha)=\sum\left(c f_{i} \log x_{i}\right)+n(c+1) \log \theta-n \theta \bar{x}+\sum f_{i}\left(\alpha+\theta x_{i}\right)-n \log \Gamma(c+1)\right)-n \log (\alpha+c+1)
$$

The three log likelihood equations are thus obtained as

$$
\begin{aligned}
& \frac{\partial \log L}{\partial \theta}=\frac{n(c+1}{\theta} \cdot+\frac{\sum f_{i} x_{i}}{\alpha+\theta x_{i}}-n \bar{x}=0 \\
& \frac{\partial \log L}{\partial c}=\frac{\sum f_{i} \log x l i}{1}+n \log \theta-\frac{n}{\alpha+c+1}-n \log (c+1)+\frac{n}{2(c+1)}=0 \\
& \frac{\partial \log L}{\partial \alpha}=\frac{\sum f_{i}}{\alpha+\theta x_{i}}-\frac{n}{\alpha+c+1}=0
\end{aligned}
$$

The three equations (7.1), (7.2) and (7.3) don't seem to be solved directly. However the Fisher's scoring method can be applied to solve these equations. We have

$$
\begin{aligned}
& \frac{\partial^{2} \log L}{\partial \theta^{2}}=-\frac{n(c+1}{\theta^{2}} \cdot+\frac{\sum f_{i} x_{i}^{2}}{\left(\alpha+\theta x_{i}\right)^{2}} \\
& \frac{\partial^{2} \log L}{\partial \alpha^{2}}=\frac{n}{(\alpha+c+1)^{2}} \cdot-\frac{\sum f_{i}}{\left(\alpha+\theta x_{i}\right)^{2}} \\
& \frac{\partial^{2} \log L}{\partial c^{2}}=\frac{n}{(\alpha+c+1)^{2}}-\frac{n}{(c+1)}-\frac{n}{2(c+1)^{2}} \\
& \frac{\partial^{2} \log L}{\partial \theta \partial \alpha}=-\frac{\partial^{2} \log L}{\left(\alpha+\theta x_{i}\right)^{2}} ; \quad \frac{n}{\partial \theta \partial c} ; \quad \frac{\partial^{2} \log L}{\partial \alpha \partial c}=\frac{n}{(\alpha+c+1)^{2}}
\end{aligned}
$$

The following equations for $\hat{\theta}, \hat{\alpha}$ and $\hat{c}$ can be solved as 
$\left[\begin{array}{lll}\frac{\partial^{2} \log L}{\partial \theta^{2}} & \frac{\partial^{2} \log L}{\partial \theta \partial \alpha} & \frac{\partial^{2} \log L}{\partial \theta \partial c} \\ \frac{\partial^{2} \log L}{\partial \alpha \partial \theta} & \frac{\partial^{2} \log L}{\partial \alpha^{2}} & \frac{\partial^{2} \log L}{\partial \alpha \partial c} \\ \frac{\partial^{2} \log L}{\partial c \partial \theta} & \frac{\partial^{2} \log L}{\partial c \partial \alpha} & \frac{\partial^{2} \log L}{\partial c^{2}}\end{array}\right]_{\substack{\hat{\theta}=\theta_{0} \\ \hat{\alpha}=\alpha_{0} \\ \hat{c}=c_{0}}} \quad\left[\begin{array}{l}\hat{\theta}-\theta_{0} \\ \hat{\alpha}-\alpha_{0} \\ \hat{c}-c_{0}\end{array}\right]=\left[\begin{array}{l}\frac{\partial \log L}{\partial \theta} \\ \frac{\partial \log L}{\partial \alpha} \\ \frac{\partial \log L}{\partial c}\end{array}\right]$,

where $\theta_{0}, \alpha_{0}$ and $c_{0}$ are the initial values of $\theta, \alpha$ and $c$ respectively. These equations are solved iteratively till sufficiently close values of $\hat{\theta}, \hat{\alpha}$ and $\hat{c}$ are obtained.

\subsection{Simulation Study of ML estimators of WQLD}

In this section, we study the performance of ML estimators for different sample sizes ( $\mathrm{n}=25,75,100,150,200,300)$. We have employed the inverse CDF technique for data simulation for WQLD using R software. The process was repeated 1000 times for calculation of bias, variance and MSE as given values in table 1. For two parameter combinations of WQLD, decreasing trend is being observed in average bias, variance and MSE as we increase the sample size. Hence, the performance of ML estimators is quite well, consistent in case of WQLD.

Table 1: Simulation Study of ML estimators for weighted Quasi Lindley Distribution

\begin{tabular}{|c|c|c|c|c|c|c|c|}
\hline \multirow{2}{*}{ Parameter } & \multirow{2}{*}{$\mathrm{n}$} & \multicolumn{3}{|c|}{$c=1.2, \alpha=0.8, \theta=0.5$} & \multicolumn{3}{|c|}{$c=2.3, \alpha=1.5, \theta=0.9$} \\
\hline & & Bias & Variance & MSE & Bias & Variance & MSE \\
\hline$\alpha$ & \multirow{3}{*}{25} & 0.698985 & 0.111666 & 0.600246 & 0.794085 & 0.331057 & 0.961628 \\
\hline$\beta$ & & 1.474035 & 0.111255 & 2.284034 & 1.2268 & 0.351011 & 1.856049 \\
\hline$\theta$ & & -0.03908 & 0.013196 & 0.014723 & 0.288817 & 0.010859 & 0.094274 \\
\hline$\alpha$ & \multirow{3}{*}{75} & 0.453829 & 0.020782 & 0.226743 & 0.412148 & 0.026023 & 0.195889 \\
\hline$\beta$ & & 1.125111 & 0.101674 & 1.367549 & 0.78452 & 0.573856 & 1.189328 \\
\hline$\theta$ & & -0.03658 & 0.012579 & 0.013917 & 0.207594 & 0.005101 & 0.048196 \\
\hline$\alpha$ & \multirow{3}{*}{100} & 0.415148 & 0.0168 & 0.189148 & 0.33123 & 0.012468 & 0.122181 \\
\hline$\beta$ & & 0.739167 & 0.094289 & 0.640657 & 0.602663 & 0.216566 & 0.579769 \\
\hline$\theta$ & & -0.05377 & 0.010805 & 0.013696 & 0.182033 & 0.004131 & 0.037267 \\
\hline$\alpha$ & \multirow{3}{*}{150} & 0.400494 & 0.011392 & 0.171787 & 0.292492 & 0.007101 & 0.092653 \\
\hline$\beta$ & & 0.69953 & 0.049215 & 0.538557 & 0.439972 & 0.128085 & 0.32166 \\
\hline$\theta$ & & -0.07008 & 0.00601 & 0.010921 & 0.151623 & 0.00527 & 0.02826 \\
\hline$\alpha$ & \multirow{3}{*}{200} & 0.21848 & 0.009064 & 0.056798 & 0.21823 & 0.005912 & 0.053536 \\
\hline$\beta$ & & 0.630678 & 0.015107 & 0.412862 & 0.225452 & 0.056483 & 0.107311 \\
\hline$\theta$ & & -0.07488 & 0.004829 & 0.010436 & 0.111407 & 0.003061 & 0.015472 \\
\hline$\alpha$ & \multirow{3}{*}{300} & 0.165541 & 0.00728 & 0.034684 & 0.138878 & 0.003637 & 0.022924 \\
\hline$\beta$ & & 0.513483 & 0.006986 & 0.270651 & 0.180969 & 0.053695 & 0.086445 \\
\hline$\theta$ & & -0.05221 & 0.000143 & 0.002869 & 0.102064 & 0.002081 & 0.012498 \\
\hline
\end{tabular}




\section{MODEL COMPARISON BASED ON SIMULATED DATA FROM WQLD}

In order to compare the Weighted Model with the base model on the basis of simulated data. In this section, we proceed by simulating a data from WQLD using inverse CDF technique discussed in the section 5.4. Two sets of random parameter combinations with sample sizes $(n=10,25,100,300,500)$ have been taken into consideration for data generation. It is evident from the table 2 and table 3, that weighted parameter plays a highly significant role for large samples. Log-likelihood ratio test reveals that the role of weighted parameter exhibits a highly significant role in case of large samples only. LR statistic for testing $H_{0}$ versus $H_{1}$ is $\psi=2\left(L(\hat{\Theta})-L\left(\hat{\Theta}_{0}\right)\right)$, where $\hat{\Theta}$ and $\hat{\Theta}_{0}$ are the MLEs under $H_{1}$ and $H_{0}$. The statistic $\psi$ is asymptotically (as $n \rightarrow \infty$ ) distributed as $\chi_{k}^{2}$, with $\mathrm{k}$ degrees of freedom which is equal to the difference in dimensionality of $\hat{\Theta}$ and $\hat{\Theta}_{0}$. $H_{0}$ will be rejected if the LR-test p-value is <0.01 (or LR Statistic value $>6.635$ ) at 99\% confidence level.

Table 2: Model Comparison Based On Simulated Data from WQLD.

\begin{tabular}{|c|c|c|c|c|c|c|}
\hline \multicolumn{4}{|c|}{$\hat{c}=0.8, \hat{\alpha}=0.1, \hat{\theta}=0.2$} & \multicolumn{2}{|c|}{ Parameter Estimates } & \multirow{2}{*}{$\begin{array}{c}\text { Likelihood } \\
\text { Ratio } \\
\text { Statistic }\end{array}$} \\
\hline Criterion & WQLD & QLD & $\begin{array}{l}\text { Sample } \\
\text { Size (n) }\end{array}$ & WQLD & QED & \\
\hline$-\log L$ & 33.14778 & 33.32745 & \multirow{4}{*}{10} & \multirow{4}{*}{$\begin{array}{c}\hat{c}=1.24(0.97) \\
\hat{\alpha}=0.96(0.87) \\
\hat{\theta}=0.213(0.06)\end{array}$} & \multirow{4}{*}{$\begin{aligned} \hat{\alpha} & =0.10(1.25) \\
\hat{\theta} & =0.16(0.89)\end{aligned}$} & \multirow{4}{*}{0.359} \\
\hline AIC & 72.29556 & 70.65489 & & & & \\
\hline AICC & 76.29556 & 72.36918 & & & & \\
\hline $\mathrm{BIC}$ & 73.20331 & 71.26006 & & & & \\
\hline$-\log L$ & 85.35855 & 87.47477 & \multirow{4}{*}{25} & \multirow{4}{*}{$\begin{array}{l}\hat{c}=1.86(1.78) \\
\hat{\alpha}=1.37(0.847) \\
\hat{\theta}=0.23(0.06)\end{array}$} & \multirow{4}{*}{$\begin{array}{l}\hat{\alpha}=0.10(0.98) \\
\hat{\theta}=0.12(0.012)\end{array}$} & \multirow{4}{*}{4.232} \\
\hline AIC & 176.7171 & 178.9495 & & & & \\
\hline AICC & 177.86 & 179.495 & & & & \\
\hline $\mathrm{BIC}$ & 180.3737 & 181.3873 & & & & \\
\hline$-\log L$ & 345.7888 & 350.2193 & \multirow{4}{*}{100} & \multirow{4}{*}{$\begin{array}{c}\hat{c}=1.20(0.57) \\
\hat{\alpha}=0.78(0.65) \\
\hat{\theta}=0.20(0.03)\end{array}$} & \multirow{4}{*}{$\begin{array}{l}\hat{\alpha}=0.11(1.52) \\
\hat{\theta}=0.13(0.07)\end{array}$} & \multirow{4}{*}{8.861} \\
\hline AIC & 697.5776 & 704.4386 & & & & \\
\hline $\mathrm{AICC}$ & 697.8276 & 704.5623 & & & & \\
\hline $\mathrm{BIC}$ & 705.3931 & 709.6489 & & & & \\
\hline$-\log L$ & 994.6124 & 1012.538 & \multirow{4}{*}{300} & \multirow{4}{*}{$\begin{array}{l}\hat{c}=1.56(0.60) \\
\hat{\alpha}=1.39(0.87) \\
\hat{\theta}=0.25(0.02)\end{array}$} & \multirow{4}{*}{$\begin{array}{l}\hat{\alpha}=0.10(0.54) \\
\hat{\theta}=0.15(0.003)\end{array}$} & \multirow{4}{*}{35.851} \\
\hline AIC & 1995.225 & 2029.076 & & & & \\
\hline AICC & 1995.306 & 2029.116 & & & & \\
\hline BIC & 2006.336 & 2036.483 & & & & \\
\hline$-\log L$ & 1694.878 & 1719.592 & \multirow{4}{*}{500} & \multirow{4}{*}{$\begin{array}{l}\hat{c}=0.87(0.23) \\
\hat{\alpha}=0.06(0.13) \\
\hat{\theta}=0.21(0.01)\end{array}$} & \multirow{4}{*}{$\begin{array}{l}\hat{\alpha}=0.12(0.46) \\
\hat{\theta}=0.14(0.0007)\end{array}$} & \multirow{4}{*}{49.428} \\
\hline AIC & 3395.757 & 3443.185 & & & & \\
\hline AICC & 3395.805 & 3443.209 & & & & \\
\hline BIC & 3408.4 & 3451.614 & & & & \\
\hline
\end{tabular}


Table 3: Model Comparison Based On Simulated Data from WQLD.

\begin{tabular}{|c|c|c|c|c|c|c|}
\hline \multicolumn{4}{|c|}{$\hat{c}=1.5, \hat{\alpha}=1.2, \hat{\theta}=1.3$} & \multicolumn{2}{|c|}{ Parameter Estimates } & \multirow{2}{*}{$\begin{array}{c}\text { Likelihood } \\
\text { Ratio } \\
\text { Statistic }\end{array}$} \\
\hline Criterion & WQLD & QLD & $\begin{array}{l}\text { Sample } \\
\text { Size (n) }\end{array}$ & WQLD & QED & \\
\hline$-\log L$ & 18.90418 & 18.96473 & \multirow{4}{*}{10} & \multirow{4}{*}{$\begin{array}{l}\hat{c}=0.549(1.12) \\
\hat{\alpha}=0.821(1.39) \\
\hat{\theta}=0.812(0.335)\end{array}$} & \multirow{4}{*}{$\begin{array}{c}\hat{\alpha}=0.10(0.45) \\
\hat{\theta}=0.70(0.21)\end{array}$} & \multirow{4}{*}{0.1210} \\
\hline AIC & 43.80836 & 41.92945 & & & & \\
\hline AICC & 47.80836 & 43.64374 & & & & \\
\hline BIC & 44.71612 & 42.53462 & & & & \\
\hline$-\log L$ & 44.00957 & 44.60075 & \multirow{4}{*}{25} & \multirow{4}{*}{$\begin{array}{l}\hat{c}=1.03(1.02) \\
\hat{\alpha}=1.18(1.01) \\
\hat{\theta}=1.04(0.28)\end{array}$} & \multirow{4}{*}{$\begin{array}{c}\hat{\alpha}=0.13(0.69) \\
\hat{\theta}=0.75(0.05)\end{array}$} & \multirow{4}{*}{1.1823} \\
\hline AIC & 94.01913 & 93.2015 & & & & \\
\hline AICC & 95.16199 & 93.74695 & & & & \\
\hline $\mathrm{BIC}$ & 97.67576 & 95.63925 & & & & \\
\hline$-\log \mathrm{L}$ & 174.5136 & 180.4392 & \multirow{4}{*}{100} & \multirow{4}{*}{$\begin{array}{c}\hat{c}=1.49(0.65) \\
\hat{\alpha}=1.21(0.985) \\
\hat{\theta}=1.18(0.163)\end{array}$} & \multirow{4}{*}{$\begin{array}{c}\hat{\alpha}=0.11(0.41) \\
\hat{\theta}=0.72(0.008)\end{array}$} & \multirow{4}{*}{11.851} \\
\hline AIC & 355.0273 & 364.8784 & & & & \\
\hline AICC & 355.2773 & 365.0022 & & & & \\
\hline BIC & 362.8428 & 370.0888 & & & & \\
\hline$-\log L$ & 517.4113 & 535.7299 & \multirow{4}{*}{300} & \multirow{4}{*}{$\begin{array}{l}\hat{c}=1.46(0.54) \\
\hat{\alpha}=0.904(0.89) \\
\hat{\theta}=1.21(0.108)\end{array}$} & \multirow{4}{*}{$\begin{aligned} \hat{\alpha} & =0.10(0.38) \\
\hat{\theta} & =0.73(0.009)\end{aligned}$} & \multirow{4}{*}{36.637} \\
\hline AIC & 1040.823 & 1075.46 & & & & \\
\hline AICC & 1040.904 & 1075.5 & & & & \\
\hline BIC & 1051.934 & 1082.867 & & & & \\
\hline$-\log L$ & 858.2261 & 892.628 & \multirow{4}{*}{500} & \multirow{4}{*}{$\begin{array}{l}\hat{c}=1.47(0.31) \\
\hat{\alpha}=0.39(0.48) \\
\hat{\theta}=1.21(0.08)\end{array}$} & \multirow{4}{*}{$\begin{array}{l}\hat{\alpha}=0.12(0.13) \\
\hat{\theta}=0.721(0.001)\end{array}$} & \multirow{4}{*}{68.803} \\
\hline AIC & 1722.452 & 1789.256 & & & & \\
\hline AICC & 1722.501 & 1789.28 & & & & \\
\hline BIC & 1735.096 & 1797.685 & & & & \\
\hline
\end{tabular}

\section{APPLICATIONS OF WEIGHTED QUASI LINDLEY DISTRIBUTIONS}

Here we analyze the strength data, reported by Badar and Priest (1982), using the Weighted Quasi Lindley Distribution (WQLD) in comparison with Quasi Lindley Distribution (QLD). Estimates of the unknown parameters is carried out in R software along with calculation of model comparison criterion values like AIC, AICC and BIC values. It may be noted that Raqab et al. (2008) fitted the 3-parameter generalized exponential distribution to the same data set. Badar and Priest (1982) reported strength data measured in GPA for single carbon fibre and impregnated 1000 carbon fibre tows. Single fibres were tested at gauge lengths of 10, 20 and $50 \mathrm{~mm}$. Impregnated tows of 1000 fibres were tested at gauge lengths of 20, 50, 150 and $300 \mathrm{~mm}$. The transformed data sets that were considered by Raqab and Kundu (2005) are used here. Table 4, data set 1 (of size 69) and table 5, data set 2 (of size 63) correspond to single fibre with $20 \mathrm{~mm}$ and $10 \mathrm{~mm}$ of gauge length, respectively. 
Table 4: Data set 1

\begin{tabular}{|l|l|l|l|l|l|l|l|l|l|}
\hline 0.031 & 0.314 & 0.479 & 0.552 & 0.700 & 0.803 & 0.861 & 0.865 & 0.944 & 0.958 \\
\hline 0.966 & 0.977 & 1.006 & 1.021 & 1.027 & 1.055 & 1.063 & 1.098 & 1.140 & 1.179 \\
\hline 1.224 & 1.240 & 1.253 & 1.270 & 1.272 & 1.274 & 1.301 & 1.301 & 1.359 & 1.382 \\
\hline 1.382 & 1.426 & 1.434 & 1.435 & 1.478 & 1.490 & 1.511 & 1.514 & 1.535 & 1.554 \\
\hline 1.566 & 1.570 & 1.586 & 1.629 & 1.633 & 1.642 & 1.648 & 1.684 & 1.697 & 1.726 \\
\hline 1.770 & 1.773 & 1.800 & 1.809 & 1.818 & 1.821 & 1.848 & 1.880 & 1.954 & 2.012 \\
\hline 2.067 & 2.084 & 2.090 & 2.096 & 2.128 & 2.233 & 2.433 & 2.585 & 2.585 & \\
\hline
\end{tabular}

Table 5: Data set 2

\begin{tabular}{|r|r|r|r|r|r|r|r|r|r|}
\hline 0.101 & 0.332 & 0.403 & 0.428 & 0.457 & 0.55 & 0.561 & 0.596 & 0.597 & 0.645 \\
\hline 0.954 & 0.674 & 0.718 & 0.722 & 0.725 & 0.732 & 0.775 & 0.814 & 0.816 & 0.818 \\
\hline 0.824 & 0.859 & 0.875 & 0.938 & 0.94 & 1.056 & 1.117 & 1.128 & 1.137 & 1.137 \\
\hline 1.177 & 1.196 & 1.23 & 1.325 & 1.339 & 1.345 & 1.42 & 1.423 & 1.435 & 1.443 \\
\hline 1.464 & 1.472 & 1.494 & 1.532 & 1.546 & 1.577 & 1.608 & 1.635 & 1.693 & 1.701 \\
\hline 1.737 & 1.754 & 1.762 & 1.828 & 2.052 & 2.071 & 2.086 & 2.171 & 2.224 & 2.227 \\
\hline 2.425 & 2.595 & 3.22 & & & & & & & \\
\hline
\end{tabular}

Fig. 4 and fig. 5, provides a graphical overview of the fitted distributions to a data given in table 4 and table 5 . It is evident graphically, that WQLD is providing a better and close fit to the data sets. In order to compare the two models using the AIC (Akaike information criterion) given by Akaike (1976), AICC (corrected Akaike information criterion) and BIC (Bayesian information criterion) given by Schwarz (1987). The better distribution corresponds to lesser AIC, AICC and BIC values.

$$
\mathrm{AIC}=2 \mathrm{k}-2 \log L \quad \mathrm{AICC}=\mathrm{AIC}+\frac{2 k(k+1)}{n-k-1} \text { and } \mathrm{BIC}=\mathrm{k} \log n-2 \log L
$$

where $\mathrm{k}$ is the number of parameters in the statistical model, $\mathrm{n}$ is the sample size and $-\log L$ is the maximized value of the $\log$ likelihood function under the considered model.

From table 6 and table 7, it is observed that Weighted Quasi Lindley distribution have the lesser AIC, AICC, -logL and BIC values as compared to Quasi Lindley distribution, which witness that WQLD fits better than QLD for data given in table 4 and table 5. In case of data set 1, Kolmogorov Smirnov p-value is greater than 0.05 for both WQLD and QLD but favors WQLD as it has greater $\mathrm{p}$-value as compared to QLD. In case of data set 2, QLD has non-significant p-value, hence does not fit statistically to the data set II but WQLD has Kolmogorov Smirnov p-value greater than 0.05. Hence we can conclude that the weighted Quasi Lindley distribution leads to a better fit than the Quasi Lindley distribution.

Table 6: ML estimates, -logL, AIC, AICC, BIC, KS-distance, KS p-values for fitted WQAD and QAD for data set 1.

\begin{tabular}{|c|c|c|}
\hline Distribution & Weighted Quasi Lindley & Quasi Lindley \\
\hline -logL & 56.37293 & 62.239 \\
\hline AIC & 118.74585 & 128.478 \\
\hline AICC & 119.15263 & 128.8848 \\
\hline BIC & 125.17526 & 132.7643 \\
\hline KS-Distance & 0.082484 & 0.13975 \\
\hline P-value & 0.7847 & 0.1706 \\
\hline ML Estimates & $\hat{c}=2.18$ & $\hat{\alpha}=0.001$ \\
& $\hat{\alpha}=0.93$ & $\hat{\theta}=1.582$ \\
\hline
\end{tabular}


Fig.4: Weighted Quasi Lindley Distribution Fitting in comparison w ith Quasi Lindley Distribution

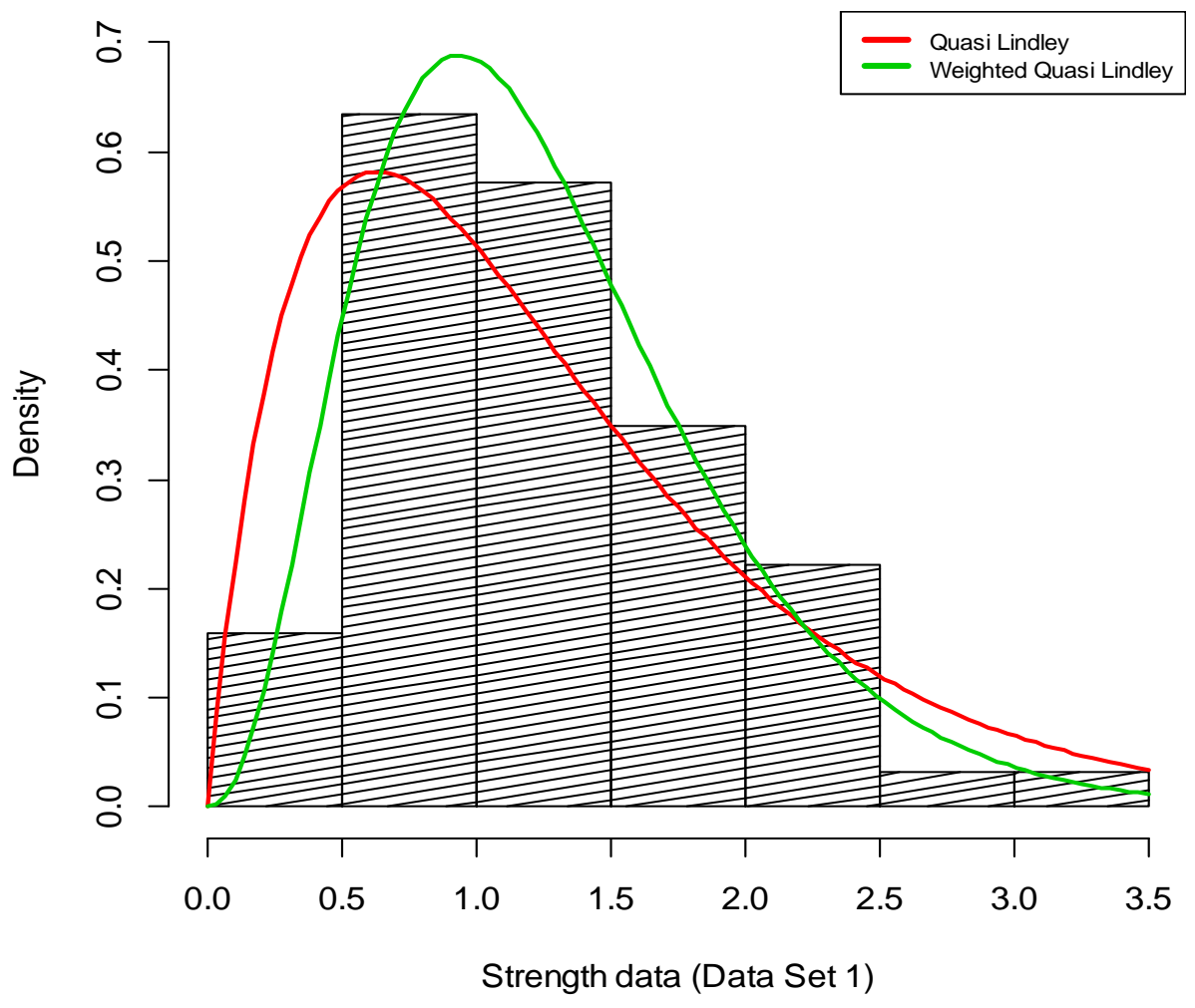

Table 7: ML estimates, -logL, AIC, AICC, BIC, KS-distance, KS p-values for fitted WQAD and QAD for data set 2.

\begin{tabular}{|c|c|c|}
\hline Distribution & Weighted Quasi Lindley & Quasi Lindley \\
\hline -logL & 62.1195 & 75.0097 \\
\hline AIC & 130.2390 & 154.0195 \\
\hline AICC & 130.6083 & 154.3887 \\
\hline BIC & 136.9413 & 158.4877 \\
\hline KS-Distance & 0.12476 & 0.25908 \\
\hline P-value & 0.233 & 0.00018 \\
\hline ML Estimates & $\hat{c}=3.32$ & $\hat{\alpha}=0.001$ \\
& $\hat{\alpha}=0.87$ & $\hat{\theta}=1.381$ \\
\hline
\end{tabular}


Fig.5: Weighted Quasi Lindley Distribution Fitting in comparison with Quasi Lindley Distribution

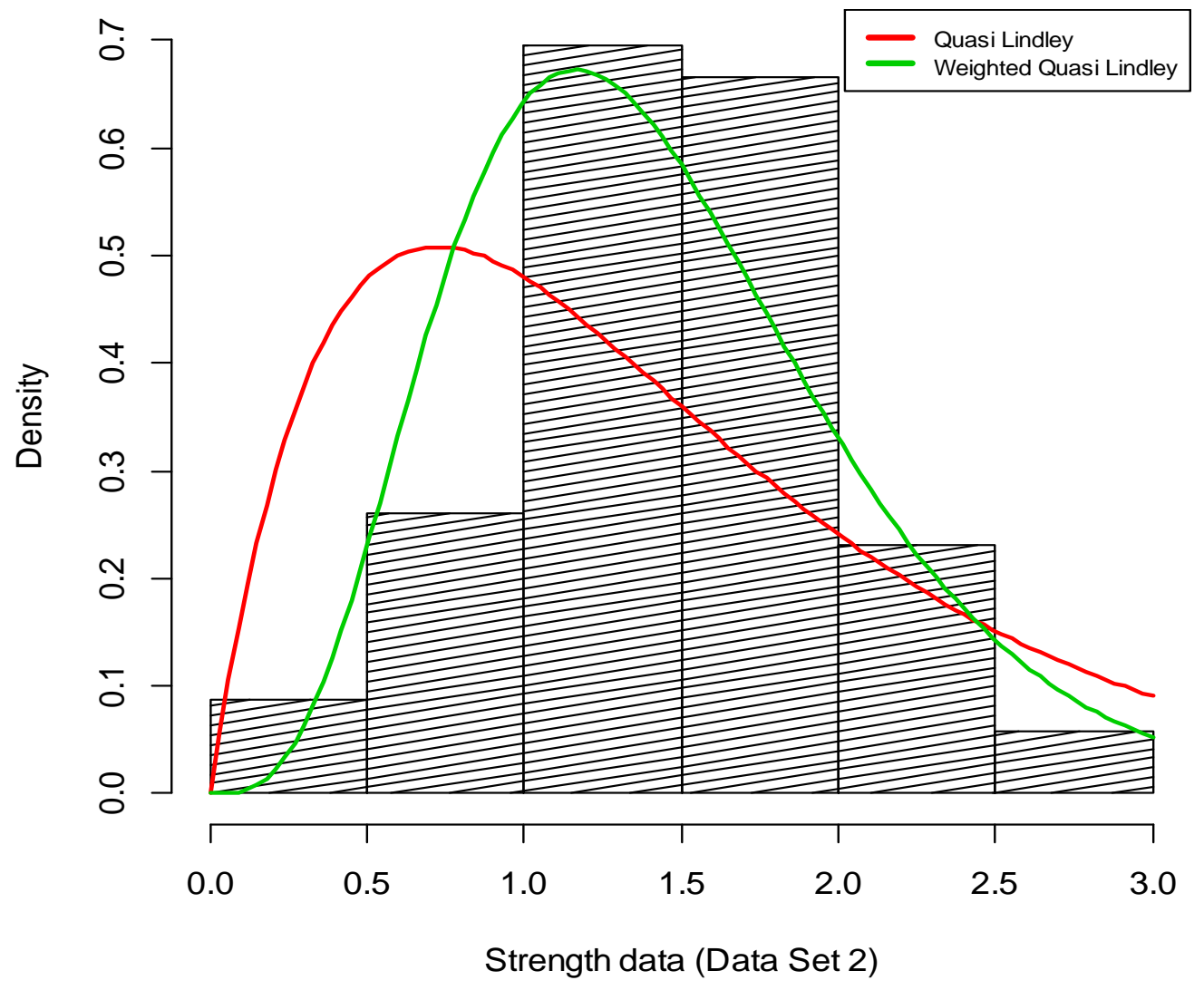

\section{CONCLUSION}

A new generalization of the Quasi Lindley distribution called the Weighted Quasi Lindley distribution has been introduced. The subject distribution is generated by using the weighting technique and taking the two parameter Quasi Lindley distribution as the base distribution. Some statistical properties along with reliability measures are discussed. Model is examined with two real life data sets for significance purpose.

\section{REFERENCES}

[1]. Akaike, H. (1976). A new look at the statistical model identification. IEEE Trans. Autom. Control , 19, $716-723$.

[2]. Bader, M. G., \& Priest, A. M. (1982). Statistical aspects of fiber and bundle strength in hybrid composites, In; hayashi T, Kawata K. Umekawa S (Eds.), Progress in Science in Engineering Composites, ICCM-IV Tokyo, 1129 -1136.

[3]. Fisher, R.A. (1934). The effects of methods of ascertainment upon the estimation of frequencies. Ann. Eugenics, 6, 13-25.

[4]. Gove, J. H. (2003). Environmental and Ecological Statistics, 10(4), 455-467. doi:10.1023/a:1026000505636

[5]. Gupta, R.C., \& Tripathi, R.C. (1996). Weighted Bivariate Logarithmic Series Distribution. Communications in Statistics-Theory and Methods, 25(5), 1099-1117.

[6]. Para, B.A., \& Jan, T. R. (2018). On three Parameter Weighted Pareto Type II Distribution: Properties and Applications in Medical Sciences. Applied Mathematics and Information Sciences Letters, 6 (1), 13-26.

[7]. Rao, C.R (1965). On discrete distributions arising out of method of ascertainment, in classical and Contagious Discrete, G.P. Patil .ed ;Pergamon Press and Statistical publishing Society, Calcutta, pp-320-332. 
[8]. Raqab, M. Z., and Kundu, D. (2005). Comparison of different estimators of $\mathrm{P}(\mathrm{Y}<\mathrm{X})$ for a scaled Burr Type $\mathrm{X}$ distribution. Communications in Statistcs - Simulation and Computation, 34, 465-483.

[9]. Schwarz, G. (1987). Estimating the dimension of a model. Ann. Stat., 5, 461-464.

[10]. Shanker, R., \& Mishra, A. (2013). A quasi Lindley distribution. African Journal of Mathematics and Computer Science Research, 6(4), 6471, DOI 10.5897/AJMCSR 12.067

[11]. Warren, W. G. (1975). Statistical Distributions in Forestry and Forest Products Research. A Modern Course on Statistical Distributions in Scientific Work, 369-384. doi:10.1007/978-94-010-1845-6_27 